\title{
International Journal of
}

\section{Information Technology and Computer Science}

\section{Kol. 9}

No. 10 Oct. 2017

\section{IJITCS Kol.9}

Http:// www.mecs-press.org

Vol.9 No.10 October 2017
Modern Education

and Computer Science PRESS 


\section{International Journal of Information Technology and Computer Science (IJITCS)}

ISSN Print: 2074-9007, ISSN Online: 2074-9015

Volume 9, Number 10, October 2017

\section{Contents}

\section{REGULAR PAPERS}

An Heuristic Approach to Solving the one-to-one Pickup and Delivery Problem with Three-dimensional 1 Loading Constraints

Rémy Dupas, Igor Grebennik, Oleksandr Lytvynenko, Oleksij Baranov

Fuzzy Based Multi-Fever Symptom Classifier Diagnosis Model

Ighoyota Ben Ajenaghughrure, P. Sujatha, Maureen I. Akazue

Data Deduplication Methods: A Review

Gagandeep Kaur, Mandeep Singh Devgan

ITAAM Framework: An Adaptive Approach to Design, Measure and Improve IT Agility

Yassine Rdiouat, Wafaa Dachry, Alami Semma

Software Product Lines Composition through Partial Derivation

Amina Guendouz, Djamal Bennouar

An Efficient Framework for Creating Twitter Mart on a Hybrid Cloud

Imran Khan, S. Kazim Naqvi, Mansaf Alam, Mohammad Najmud Doja, S. Nasir Aziz Rizvi

Ear Biometric System using GLCM Algorithm

Sajal Kumar Goel, Mrudula Meduri

Model Driven Approach for Test Data Optimization Using Activity Diagram Based On Cuckoo Search

Algorithm

Rajesh Ku. Sahoo, Durga Prasad Mohapatra, Manas Ranjan Patra 